\title{
ANTITHROMBOTIC TREATMENT FOR ATRIAL FIBRILLATION
}

\author{
Gregory Y H Lip, Gordon D O Lowe
}

\author{
Atrial fibrillation is an important risk \\ factor for stroke, and \\ antithrombotic treatment should be \\ considered in most patients with \\ this arrhythmia
}

Although atrial fibrillation has long been recognised as a risk factor for thromboembolic events, preventive treatment has, until recently, been both empirical and controversial. Only in the past few years have antithrombotic drugs been proved by prospective clinical studies to be effective against strokes in atrial fibrillation.

\section{Atrial fibrillation and thromboembolism}

\section{Non-rheumatic atrial fibrillation and stroke}

- Non-rheumatic atrial fibrillation has been associated with a fivefold increase in the risk of ischaemic stroke

- The yearly risk is $5-7 \%$, increasing with age

- Computed tomography studies have shown that silent ischaemic cerebral infarction is present in $26 \%$ of patients with non-rheumatic atrial fibrillation

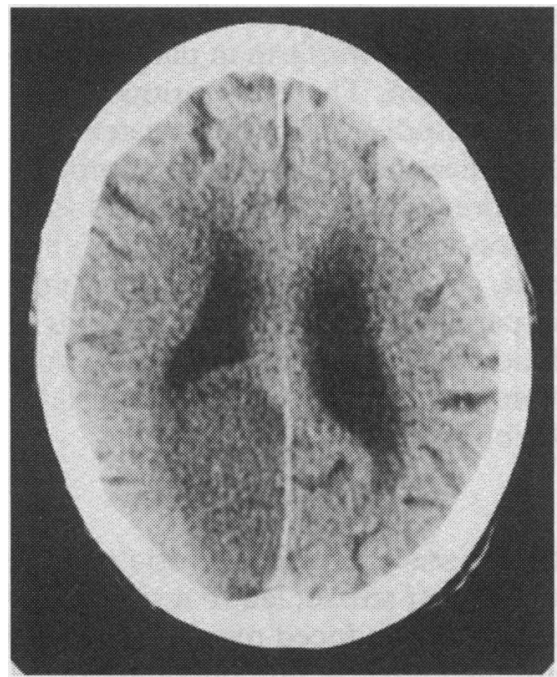

Computed tomogram showing large thrombotic stroke.
The pathophysiological mechanism for thromboembolism seems to be the disturbed blood flow in the fibrillating left atrium, which predisposes to the formation of thrombi and arterial embolism, especially in the presence of underlying heart disease. About 15-20\% of patients who have an acute stroke have atrial fibrillation at the time of their stroke; mortality is one and a half to three times higher in those patients than in patients with sinus rhythm at the time of stroke.

\section{Underlying heart disease}

Underlying heart disease-including valvar and hypertensive heart disease, an enlarged left atrium, and poor left ventricular function-is a contributory risk factor for stroke and thromboembolism in atrial fibrillation. The risk of thromboembolism in atrial fibrillation is 18 times greater if rheumatic heart disease is present. In the stroke prevention in atrial fibrillation study, the presence of recent (within three months) congestive heart failure and left ventricular dysfunction on echocardiography also contributed to an increased risk of thromboembolism in atrial fibrillation. This is consistent with the observation that thromboembolism is a common cause of death in patients with congestive heart failure, occurring in up to $30 \%$ of patients, which is partly preventable by anticoagulation.

\section{Left atrial enlargement and spontaneous echo contrast}

An enlarged left atrium may contribute to an increased risk of atrial thrombi and thromboembolism in patients with atrial fibrillation. An enlarged left atrium has also been associated with "spontaneous echo contrast" on transoesophageal echocardiography-a smoke-like appearance of blood in the atria, suggesting sluggish flow. It is associated with dilated left atria, intracardiac thrombi, thromboembolism, stroke, abnormal rheology, and coagulation.
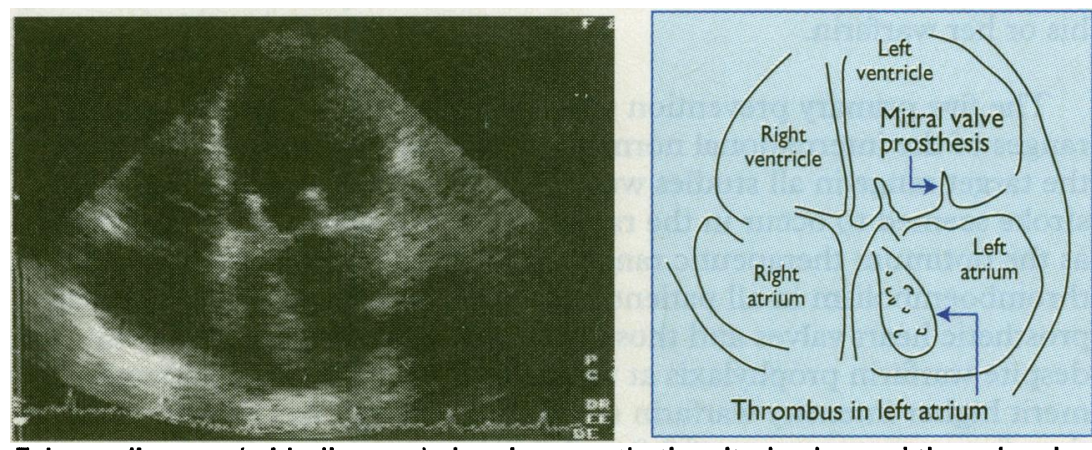

Echocardiogram (with diagram) showing prosthetic mitral valve and thrombus in left atrium.
Previous cerebrovascular disease or thromboembolism

A history of stroke, transient ischaemic attack, or other embolic events adds to the risk of stroke and mortality in atrial fibrillation. The pooled analysis by the Atrial Fibrillation Investigators showed that a previous stroke or transient ischaemic attack was an independent risk factor for further strokes. Using warfarin in such patients reduced the annual rate of stroke from $12 \%$ a year to $5 \%$. 


\section{Paroxysmal and chronic atrial fibrillation and stroke risk}

- A third of patients with paroxysmal atrial fibrillation develop chronic atrial fibrillation over two to three years

- The risk of stroke is highest during the first months after the initial diagnosis of atrial fibrillation or immediately after a transition from paroxysmal to chronic atrial fibrillation

- The recent pooled analysis by the Atrial Fibrillation Investigators suggested that patients with paroxysmal atrial fibrillation and chronic atrial fibrillation had a similar risk of stroke, although the length of time a patient was in atrial fibrillation had no discernible effect on the risk
In a Glasgow study, among patients admitted with acute severe stroke, those with atrial fibrillation (25\%) had a significantly higher hospital mortality than those in sinus rhythm (67\% v 44\%). This was confirmed in the Oxfordshire community stroke project, in which the 30 day mortality among patients with acute stroke was three times higher among those with atrial fibrillation (17\%) than among those in sinus rhythm.

\section{Hypertension and diabetes}

A history of hypertension and diabetes add to the risk of stroke in atrial fibrillation. Using warfarin in patients with a history of hypertension or diabetes would reduce the annual rate of stroke respectively from $5-6 \%$ to $2 \%$ and from $9 \%$ to $3 \%$.

\section{Duration and onset of atrial fibrillation}

The onset of atrial fibrillation may be related to the imminence of stroke. In the Framingham study, atrial fibrillation was present at the time of stroke in $24 \%$ of subjects, and about a third of the strokes associated with atrial fibrillation occurred within six months of onset of the arrhythmia. In addition, a further stroke within six months of the first may be more common in patients with continued atrial fibrillation.

Many patients with paroxysmal atrial fibrillation go on to develop chronic atrial fibrillation, and thromboembolic complications often occur during the transition. associated with the sick sinus syndrome and patients with this condition are at farticular risk of stroke and thromboembolism.

\section{Clinical trials of warfarin and aspirin prophylaxis}

\section{Important stroke prevention trials}

AFASAK Copenhagen atrial fibrillation, aspirin, anticoagulation study

BAATAF Boston area anticoagulation trial for atrial fibrillation

CAFA Canadian atrial fibrillation anticoagulation study

EAFT European atrial fibrillation trial

SPAF Stroke prevention in atrial fibrillation trial

SPINAF Stroke prevention in nonrheumatic atrial fibrillation study

\section{Warfarin}

In the mid-1980s five randomised prospective clinical trials were independently started to define the value of anticoagulant prophylaxis with warfarin in patients with atrial fibrillation. These five primary prevention studies showed consistent results. Since publication of the results another primary prevention trial (the second stroke prevention in atrial fibrillation study) has been reported, as well as a secondary prevention trial in patients with non-rheumatic atrial fibrillation and a history of transient ischaemic attack or minor stroke (European atrial fibrillation trial).

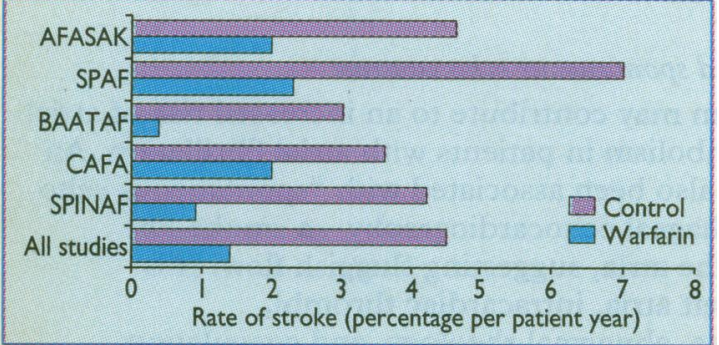

Efficacy of warfarin in atrial fibrillation trials-total risk reduction for all five trials combined is $68 \%$ $(P<0.001)$.

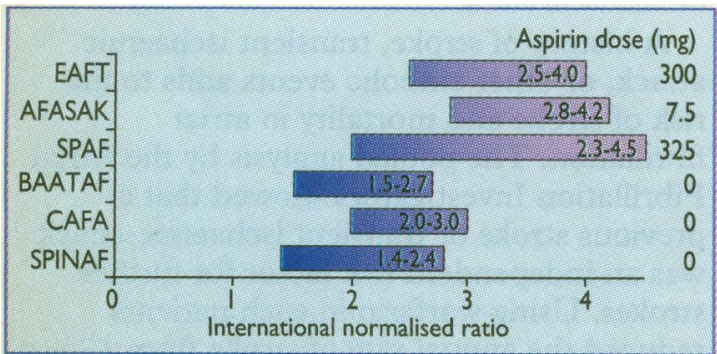

Ranges of international normalised ratios used in atrial fibrillation trials.
The pooled analysis of the five primary intervention trials showed that warfarin reduced the annual rate of stroke; led to a similar risk reduction in stroke with residual deficit; reduced mortality by a third; and reduced the rate of the combined adverse outcome (stroke, systemic embolism, or death) by half. The efficacy of warfarin prophylaxis was in fact underestimated because most strokes in patients allocated to warfarin occurred while the patient was not taking his or her warfarin.

The five primary prevention trials used different target therapeutic ranges of the international normalised ratio of the prothrombin time; the target range in all studies was 1.5 to 4.5 . The minimum risk of stroke seemed to occur in the range 2.0 to 3.0 , which is now accepted as the optimum therapeutic range for warfarin prophylaxis of thromboembolism in all patients except those with older, mechanical prosthetic heart valves and those with recurrent thromboembolism despite warfarin prophylaxis at the range 2.0 to 3.0 (such patients merit higher intensity warfarin (range 3.0 to 4.5 )). At present, therefore, a target range of 2.0 to 3.0 can be recommended in the absence of the above indications for high intensity warfarin. 
- Risk of bleeding in patients taking warfarin increases exponentially with an increase in anticoagulant effect, rising from an annual risk of $0.2 \%$ with an international normalised ratio of 2.0 to $3.0 \%$ with a ratio of 4.0

- Bleeding is most common in patients with unstable anticoagulation control and those with a history of thromboembolism and ranges from $11 \%$ to $40 \%$

- Complications related to bleeding may occur often, however, even when the international normalised ratio is within the therapeutic range

\section{Does evidence from prevention studies apply to clinical practice?}

- The patient population was highly selected-for example, $>90 \%$ of those screened in many of the studies were excluded from entry

- The rate of patient withdrawal in the studies was often high $(19 \%$ to $38 \%)$

- Patients in usual clinical practice may have different risks of thromboembolism and bleeding from selected patients participating in trials

- The compliance, and therefore safety, of warfarin may be poorer when it is used in clinical practice than when it is used in carefully selected, well motivated, and closely monitored study participants

\section{Further questions on antithrombotic treatment in atrial fibrillation}

- What is the efficacy of aspirin (which does not need laboratory monitoring and carries a lower risk of bleeding) compared with warfarin as antithrombotic prophylaxis?

- For which patients with the highest risk of thromboembolism may the risk of bleeding and the monitoring of warfarin prophylaxis be most justified?

- Are there any patients whose risk of thromboembolism is so low that neither aspirin nor warfarin prophylaxis is indicated?
Intracranial haemorrhage is the most feared complication of warfarin prophylaxis. The annual risks of intracranial haemorrhage increased from $0 \cdot 1 \%$ in the controls to $0.3 \%$ in patients taking warfarin in the pooled analysis. This risk was associated with an international normalised ratio $>3.0$ and with uncontrolled hypertension; there was also a non-significant association with increasing age. Lower intensity warfarin (for example, target ratio 1.5 to $2 \cdot 5$ ) is currently being compared with higher intensity warfarin in the third stroke prevention in atrial fibrillation study; meanwhile some physicians use low intensity warfarin in patients for whom the usual target range of 2.0 to 3.0 is judged to carry an unacceptable risk of bleeding.

Despite the convincing evidence from the pooled analysis of randomised trials that warfarin prophylaxis is highly effective and seems to outweigh substantially the risk of intracranial bleeding (as well as other types of major bleeding), the efficacy of warfarin in clinical trials may not be reproduced in practice. This is due to the highly selected patients in these studies.

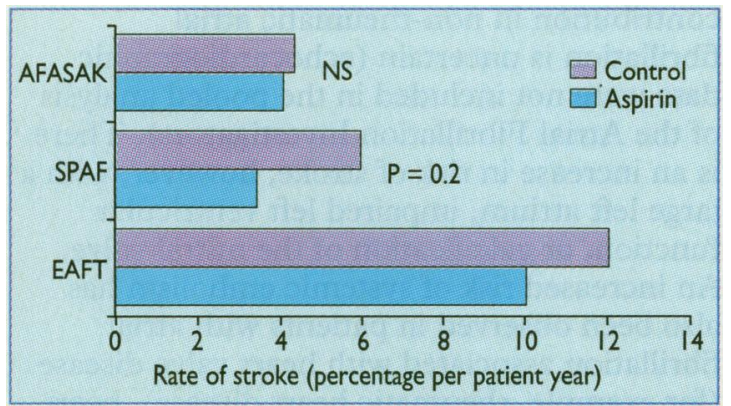

Efficacy of aspirin in atrial fibrillation trials-total risk reduction for all three trials combined is $21 \%(P=0.04)$.

\section{Aspirin}

Aspirin has been evaluated as primary prophylaxis of systemic thromboembolism - in two randomised trials compared with no antithrombotic treatment and in one compared with warfarin: aspirin was only about half as effective as warfarin, with a $36 \%$ decrease in risk of stroke. Aspirin prophylaxis had no significant effect on stroke with residual deficit or mortality; but there was a $28 \%$ decrease in the rate of the combined outcome of stroke, systemic embolism, or death.

The annual risk of stroke seems low, however, in patients at moderate risk treated with aspirin, and aspirin is simpler to monitor and has a lower risk of bleeding. Aspirin was less effective than warfarin, however, when given as secondary prophylaxis in patients with previous stroke or transient ischaemic attack, who have a higher risk of thromboembolism.
Risk of stroke in patients with atrial fibrillation, stratified by risk, with and without antithrombotic prophylaxis. Values are percentages per patient per year

\begin{tabular}{llcc}
\hline Risk & $\begin{array}{l}\text { No } \\
\text { prophylaxis }\end{array}$ & Aspirin & Warfarin \\
\hline $\begin{array}{l}\text { High: } \\
\text { Previous stroke or transient ischaemic attack } \\
\begin{array}{l}\text { Age } \geqslant 75, \text { other clinical risk factors* } \\
\text { Moderate: }\end{array}\end{array}$ & 12 & 10 & $4-5$ \\
$\begin{array}{l}\text { Age }<65, \text { other clinical risk factors* } \\
\text { Age } 65-74\end{array}$ & 8 & $4-5$ & $1-2$ \\
$\begin{array}{l}\text { Age } \geqslant 75, \text { no clinical risk factors } \\
\text { Low: } \\
\text { Age }<65, \text { no clinical risk factors }\end{array}$ & 4 & $1-2$ & $1-2$ \\
\hline
\end{tabular}

*Such as diabetes and hypertension.
Risk stratification for warfarin, aspirin, or no antithrombotic prophylaxis

The relative risk of stroke in people with non-rheumatic atrial fibrillation (and hence the absolute benefits of antithrombotic prophylaxis with warfarin or aspirin) varies greatly with the presence of risk factors for thromboembolism. The risk of stroke in nonrheumatic atrial fibrillation seems similar in men and women, in people with continuous atrial fibrillation, and in those with paroxysmal atrial fibrillation. 


\section{Identifying patients with atrial fibrillation at high risk of stroke and thromboembolism}

- Many such patients may be unrecognised and undiagnosed in the community, with few or no symptoms

- Formal screening for atrial fibrillation in primary care should be considered

- Careful clinical history is needed to identify those with important risk factors

- Echocardiography may be a further tool in thromboembolic risk stratification

- Recent studies suggest that a "hypercoagulable" state exists in patients with atrial fibrillation

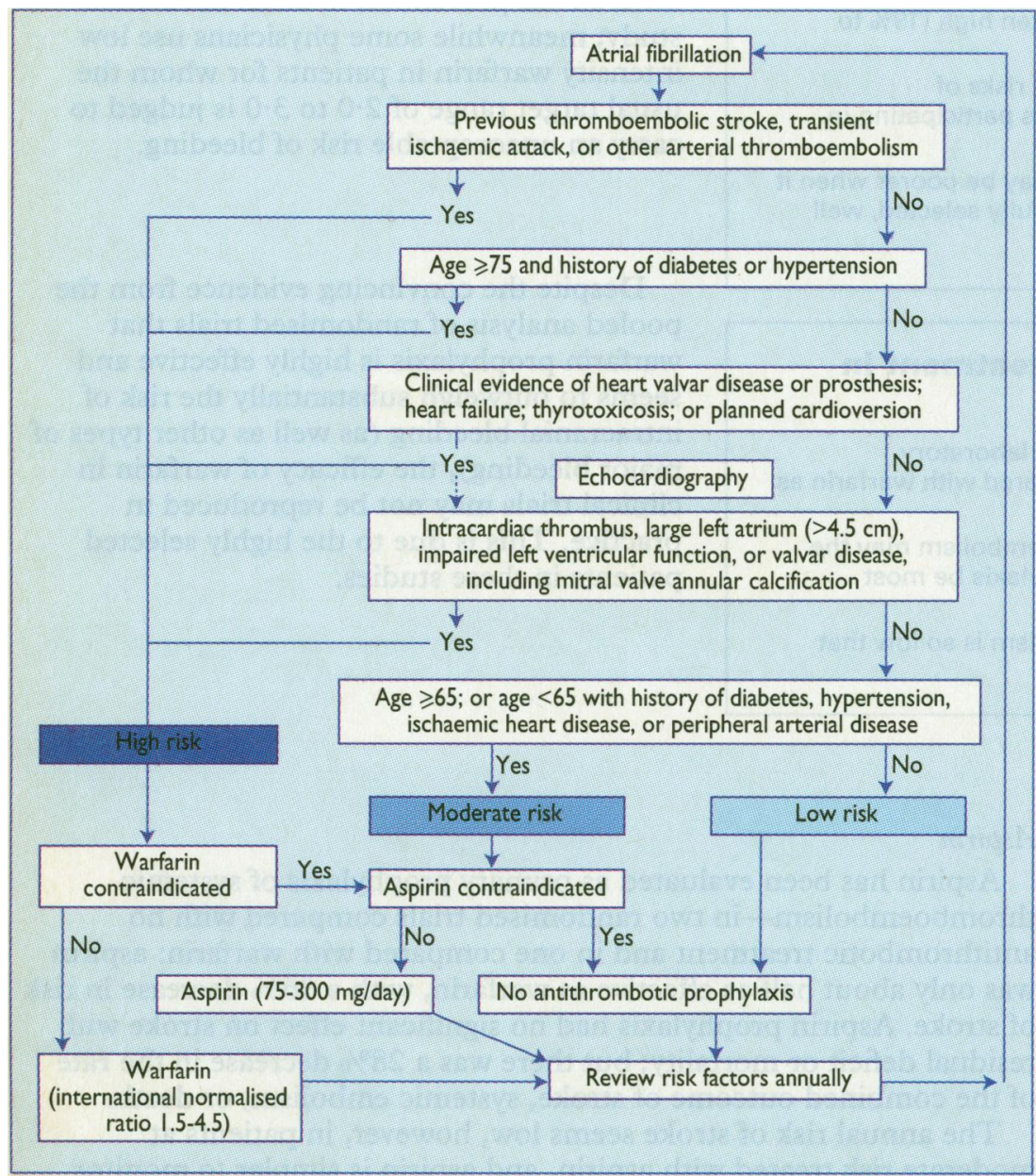

Algorithm for risk stratification and selection of prophylaxis in atrial fibrillation.

\section{Factors that may increase risk of bleeding with warfarin}

- Age

- Uncontrolled hypertension (systolic blood pressure $>180 \mathrm{~mm} \mathrm{Hg}$ or

diastolic blood pressure $>100 \mathrm{~mm} \mathrm{Hg}$ )

- Alcohol excess

- Liver disease

- Poor drug or clinic compliance

- Bleeding lesions (especially gastrointestinal blood loss-for example, peptic ulcer disease-and previous cerebral haemorrhage)

- Tendency to bleeding (including coagulation defects, thrombocytopenia)

- Concomitant use of aspirin with oral anticoagulants
The highest risk of stroke (annual risk 12\%) is in people with previous transient ischaemic attack or stroke. Warfarin is more effective in absolute terms as secondary prophylaxis than as primary prophylaxis and also seems more effective than aspirin. In addition to a history of thromboembolism, other independent risk factors for thromboembolism are increasing age (especially age $>75$ years), diabetes mellitus, and history of hypertension. These high risk patients should be considered for anticoagulation with warfarin.

A moderate risk of stroke (annual risk 4\%) exists in people with non-rheumatic atrial fibrillation, without previous embolism, who are $(a)$ aged under 65 years with a history of diabetes or hypertension; (b) aged 65-74 (with or without a history of diabetes or hypertension); or (c) aged 75 years or more with no history of diabetes or hypertension. In such people aspirin seems as effective as warfarin (reducing the annual risk of stroke to $1 \%$ to $2 \%$ ).

A low risk of stroke (annual risk 1\%) exists in people with non-rheumatic atrial fibrillation who are aged under 65 years with no history of embolism, hypertension, or diabetes. In such people antithrombotic prophylaxis with either aspirin or warfarin does not seem warranted unless some other indication coexists.

Echocardiography may complement the clinical risk stratification of risk of stroke in atrial fibrillation, although the precise contribution in non-rheumatic atrial fibrillation is uncertain (echocardiographic data were not included in the pooled analysis of the Atrial Fibrillation Investigators). There is an increase in risk of stroke, however, with a large left atrium, impaired left ventricular function, or calcification of the mitral valve. An increased risk of systemic embolism has also been observed in patients with atrial fibrillation associated with heart valve disease (for example, rheumatic heart disease), heart valve prosthesis, heart failure, intracardiac thrombus, or thyrotoxicosis. One possible algorithm for risk stratification and selection of prophylaxis in atrial fibrillation is shown.

\section{Practical considerations in antithrombotic prophylaxis}

Before starting antithrombotic prophylaxis with warfarin or aspirin it is important to balance the risks (especially bleeding) and benefits in a patient. To minimise the risk of intracranial bleeding with prophylactic warfarin, hypertension should be adequately controlled, and the risks and benefits of warfarin reviewed annually, especially in patients aged over 80 . 


\section{Recommendations by American College of Chest Physicians on starting anticoagulation after acute thromboembolic stroke}

- Patients with small or moderate thromboembolic strokes in whom an intracranial bleed is excluded by computed tomography at $\geqslant 48$ hours:

Heparin followed by warfarin (international normalised ratio $2 \cdot 0-3 \cdot 0$ )

- Patients with large embolic strokes or uncontrolled hypertension: No anticoagulation for 5-14 days because of the increased risk of haemorrhagic transformation

- Patients with non-valvar atrial fibrillation as the presumed cause of thromboembolism:

Warfarin only (after computed tomography at $\mathbf{4 8}$ hours) owing to the low risk of early recurrent thromboembolism

\section{Summary}

- Antithrombotic prophylaxis with long term warfarin or aspirin reduces thromboembolic risk in atrial fibrillation

- Identification, risk assessment, and regular review of all patients with atrial fibrillation should be routine in general and hospital practice

- Risk stratification is easily performed on clinical groundsechocardiography may refine it

\section{Key references}

Albers GW. Atrial fibrillation and stroke. Arch Intern Med 1994;154:1443-8

Atrial Fibrillation Investigators. Risk factors for stroke and efficacy of antithrombotic therapy in atrial fibrillation. Arch Intern Med

1994; 154:1449-5

British Society for Haematology. Guidelines on oral anticoagulation: second edition. J Clin Pathol 1990:43:177-83

Lip GYH. Does atrial fibrillation confer a hypercoagulable state? Lancet 1995;346:1313-4

Laupacis A, Albers GW, Dalen JE, Dunn MI, Feinberg W, Jacobson AK. Antithrombotic therapy in atrial fibrillation. Chest 1995;108(suppl):352-9S
In patients with acute stroke and atrial fibrillation, intracranial haemorrhage should therefore be excluded-for example, with computed tomography-before starting warfarin. This is important as $11 \%$ of patients with haemorrhagic stroke have atrial fibrillation, compared with $18 \%$ with stroke due to cerebral infarction. It is important to start anticoagulation at the right time after an acute cardioembolic stroke as there is the risk of haemorrhagic transformation of the cerebral infarct, by bleeding into the infarcted, softened brain.

The graphs showing effectiveness of warfarin and aspirin and the international normalised ratios, have been adapted, with permission, from Albers et al, Arch Intern Med 1994;154:1443-8. The table was adapted, with permission, from Atrial Fibrillation Investigators, Arch Intern Med 1994;154:1449-57. The recommendations in the box on when to start anticoagulation were adapted from Sherman DG et al, Chest 1995;108(suppl): 444-56S.

Gregory Y H Lip is a senior registrar in the university department of medicine and department of cardiology, City Hospital, Birmingham, and Gordon D O Lowe is professor of vascular medicine in the university department of medicine, Royal Infirmary, Glasgow.

The ABC of Atrial Fibrillation is edited by Gregory Y H Lip, currently in the department of cardiology, Walsgrave Hospital, Coventry.

\section{Correction}

\section{ABC of Atrial Fibrillation}

Authors' errors occurred in two places in the third box in the firs chapter of the ABC of Atrial Fibrillation by Gregory Y H Lip and D Gareth Beevers (18 November, pp 1361-3). Engelmann's name was misspelt, and Bouillaud [not Bouilland] made his discovery about digitalis in 1835 [not 1935].

\section{Clinical encounters in cyberspace}

We recently redesigned our hospital discharge summary and added my Internet email address. The general practitioners in our area have my telephone and fax numbers and use these frequently to contact me about our patients, and I hoped that those connected would make use of this additional facility to get in touch with me. Patients are given copies of their summaries to take home.

A few weeks ago I logged on to retrieve my mail and was surprised to find a message from a patient discharged only a week before. He had obtained my email address from his copy of the discharge summary and welcomed the chance to tell me that he was pleased with the care that he had received in hospital and was making a good recovery at home. There were, however, aspects of his care that he wanted to discuss but was embarrassed to talk about. He did not like to telephone or write and hoped that I could reply to his email address. To prove that he was indeed who he said he was, he gave me his postal address, hospital number, and telephone number. When I rang to verify recognised his voice and convinced him that confidentiality was best maintained if we talked rather than communicated by email. Twenty minutes later all his questions were answered to his satisfaction.

Another way I have been contacted by a patient is with the talk facility on the Internet. If your computer is switched on and you are logged on and have this facility invoked people on other computers can attract your attention to say that they want to talk to you. If you respond the screen on your computer splits into two and communication begins. You type on one half while the caller's message appears on the other. For those who cannot type fast there are suggested abbreviations such as BCNU (be seeing you), IMHO (in my humble opinion), and ROTFL (rolling on the floor laughing).

Cyberspace is an exciting place for information exchange but sensitive information must not be disseminated this way until there are better safeguards. The password, encrypted to ensure that only the person authorised is allowed access to an email box, is not as secure as people think. There are programs now widely available to decipher passwords. The information on the computer in your office is as secure as the lock on the door. Once you connect your computer to the Internet the files on your system can be retrieved by anyone with the right resources. If your computer is connected to a network-for instance, in a hospital-the entire information on that network is vulnerable. Abuse can also occur in other ways. Anyone who knows my email address can send messages to others in my name, and the recipient has no way of knowing whether I was the initiator. Mail in transit can be intercepted and even altered. I agree with the BMA that doctors should not cooperate with the implementation of the NHS superhighway unless the government can ensure that patients' medical records are secure. If Pentagon computers can be infiltrated by hackers almost to the point of starting a nuclear war what chance has the cash strapped NHS? The electronic password system will go down in history as one of the least innovative ways of protecting access to computer data.-DAVID SELLU is a senior lecturer in surgery in London, and his email address is david sellu@sellutec.demon.co.uk 